\title{
ON QUALITY ISSUES IN NETWORKED VALUE CONSTELLATIONS
}

\author{
Novica Zarvic ${ }^{*}$, Roel Wieringa, Pascal van Eck \\ Information Systems Group, Department of Computer Science, \\ University of Twente, THE NETHERLANDS \\ \{n.zarvic,r.j.wieringa, p.a.t.vaneck\}@ewi.utwente.nl
}

\begin{abstract}
One of the main purposes of collaborative networks is to satisfy specific consumer needs, which one company cannot satisfy alone. With the opening of the internet in the 1990s the number of companies that collaborate by means of computer networks increased rapidly. As far as one of our main foci is the consideration of value object exchanges between the involved business actors, we refer to such collaborative networks as networked value constellations or value webs. The business requirements of networked value constellations need to be enabled and operationalized by means of functional and quality requirements at the IT level. Our paper aims to build a sound understanding of how to plan quality related issues by considering distinct perspectives, namely the business perspective and the information systems perspective. Each perspective requires multiple quality-related considerations. From a business perspective, we have (a) to consider the quality perceptions by the end consumers, (b) to plan the quality of the value objects to be produced, and (c) to plan the quality of the value objects to be transferred. From an information systems perspective we need (d) to plan the quality of the software-intensive systems. The last quality issue, (e) structural properties of the network, has to be applied to both mentioned perspectives. In this paper we provide a framework for discussing and addressing the described quality issues and suggest several techniques in doing so. We point out where these techniques can e used as such and where additional research is required.
\end{abstract}

\section{INTRODUCTION}

A value web or a networked value constellation represents an inter-organizational business setting by considering value exchanges between independent business actors. $e^{3}$-value models (Gordijn, 2001; Gordijn, 2003), as a graphical representation form of value webs, are based on principle of economic reciprocity. This means that for every value exchange, something of value is expected in return. The $e^{3}$-value approach was introduced in a time when many dot-coms failed financially, because many companies wished for a 'slice of the cake' without assessing the economic sustainability of the business idea as a whole. In recent work, we have shown how to check functional alignment between value models and the supporting IT functionality (Zarvić, 2008). We are of the opinion that, next to the financial assessment of possible future success and pure functional alignment, also diverse

\footnotetext{
* Supported by the Netherlands Organisation for Scientific Research (NWO), project 638.003.407 (Value-based Business-IT Alignment)
} 
quality issues play an important, if not crucial, role in the overall success. In this analytical work, we discuss important quality-related considerations that arise during the business-IT alignment task of networked value constellations, of which some are resolved and some not. Thereby we distinguish between two different perspectives, namely the business perspective and the information systems (IS) perspective. In section 2 we present our alignment framework, a conceptual framework showing the main relation between the perspectives, before we present an illustrative $e^{3}$-value example. On the basis of this example we discuss in the remaining sections the identified quality issues along the two perspectives, suggest several techniques for this setting, and point out how they are related, before we draw our conclusions.

\section{CONSIDERING QUALITY IN E-BUSINESSES}

\subsection{Quality issues and the alignment framework}

The design of a system that supports a business need is a complex process with many different stakeholders involved. For managing this complexity, researchers apply multi-perspective approaches. In this paper we distinguish the business perspective and the IS perspective. The two are interrelated and their main relationship is a "put into operation" relation (Gordijn, 2003), which means that the IS perspective enables the previously stated business requirements.

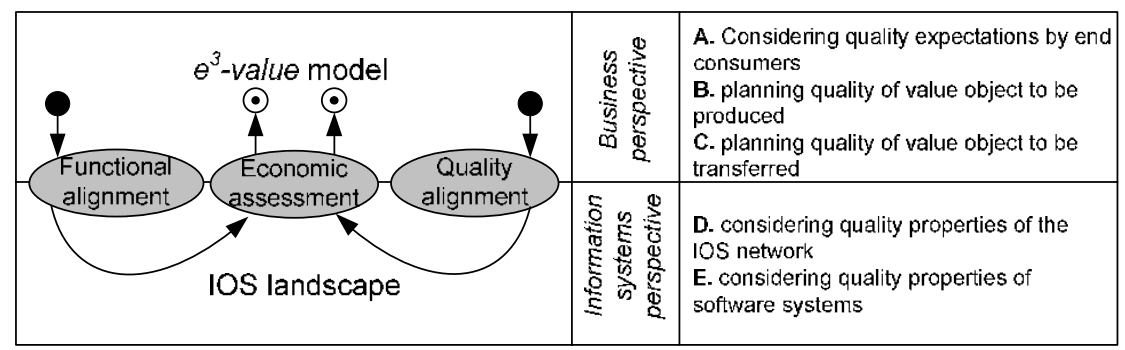

Figure 1 - Multi-perspective quality issues in the context of Networked business-IT alignment

Figure 1 shows the two perspectives under consideration and represents on the left our alignment framework for value webs. Firstly, on the basis of the value-based business requirements, designers need to elicitate the functional requirements of the underlying IS level. This process might reveal the need for completely new systems that need to be build or even bought by the respective business actors. Of course such investments must subsequently be considered in the profitability calculations. Secondly, we need to identify at what level of quality the business requirements captured in $e^{3}$-value models should be enabled. If we have implemented an epayment mechanism, and this mechanism is neither secure nor fast enough, we cannot assume this leading to a business success story. Quality is defined by ISO 8402 to be "the totality of characteristics of an entity that bear on its ability to satisfy stated and implied needs" (ISO, 1994). Taking this general definition, we can identify in our alignment framework on the right of Figure 1 several locations along the two perspectives, where we deal with such quality characteristics: 
A. quality expectations of end consumers (business perspective),

B. quality planning during value object composition/production (business perspective),

C. quality properties of the business network (business perspective),

D. quality properties of the IOS network (information systems perspective),

E. quality properties of software systems (information systems perspective).

The elicitation of quality requirements in the context of networked value constellations is, to the best of our knowledge, insufficiently addressed in the literature. Derzsi (2007) addresses scalability issues in value webs by relating the number of value object transfers to the number of invocations in an UML deployment diagram, but there exists no work that encompasses and structures the distinct quality issues between the two perspectives in a value web context, like done in this paper.

\subsection{An example business case}

In the following we will introduce the $e^{3}$-value modeling notation, before we treat in the coming sections each quality issue separately. Figure 2 shows an $e^{3}$-value model, in which end consumers purchase a value object $a$, which satisfies their needs, from a retailer, but the retailer will not work for free and expects something of value in return, i.e. value object $b$.

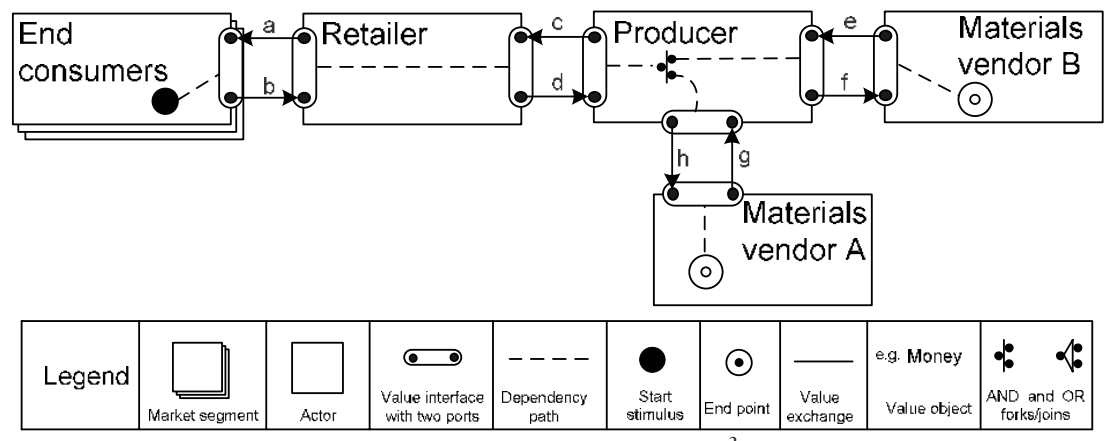

Figure 2 - Educational example of an $e^{3}$-value model.

O'Sullivan (2002) describes this as the obligation to pay for a service. Such a refundment obligation does not necessarily consist of money or fees in the classical sense, but it can be any object of economic value. The retailer gets the value object from a producer, who is composing/producing the value object by combining value objects, as the ingredients of the object to be produced, from diverse vendors.

The concepts used are: an actor is a participant in the value web and an independent, rational economic entity. A market segment is a group of actors that share the same needs. Actors exchange value objects with each other. A value object is anything that is of value for at least one actor or market segment, such as money, a service, a product, or an experience. The $e^{3}$-value principle of economic reciprocity is hereby assumed, so that a transfer of a value object is always coupled to a reciprocal value transfer. Value objects are transferred through value ports. Value interfaces are groupings of value ports. A value exchange between two actors, then, connects two value ports with each other and represents an atomic trade of 
value objects between value ports. Value activities can be assigned to actors and represent a collection of operational activities, which must yield profit. To show which value exchanges are needed to fulfill a consumer need, we can draw a dependency path, which is a set of connected line segments that starts with a filled circle (representing the occurrence of a the consumer need) and ends in a double lined circle (representing the boundary of our model). AND/OR elements can be used for merging and splitting parts of a dependency path.

\section{QUALITY ISSUES AT BUSINESS LEVEL}

\subsection{End Consumer Quality Expectation of Value Object Consumption}

Quality issue A, the expectations of the end consumers on the quality of the value object to be consumed is one of the most important locations to look at while planning inter-organizational business constellations, because a value web exists by definition for satisfying complex needs of end consumers.

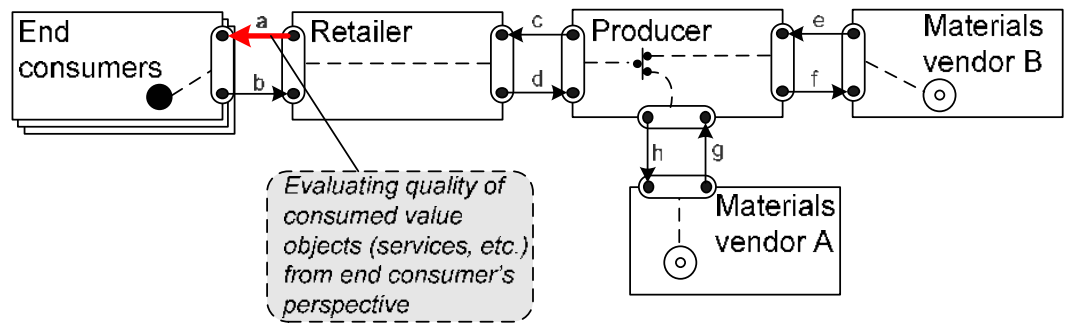

Figure 3 - Planning End Consumer Quality Perception in a Value Web

In the service marketing literature end consumer expectations and perceptions on service quality are measured by means of the SERVQUAL model (Parasuraman, 1985). The original SERVQUAL model considers 10 quality determinants, including well known quality attributes as reliability, security, responsiveness, understandability, etc. In previous research we showed the main conceptual relations of these determinants to software quality characteristics as suggested by the ISO 9126 standard (Zarvić, 2007). SERVQUAL considers quality to be the difference between customer expectations and perceptions, and is considered to be a measure of how well the service level delivered matches customer expectations. For determining this difference, the so-called SERVQUAL questionnaire, a 22-items likert scale, is used. By considering quality as the difference between expectation and perceived delivery, the results of this analysis are mainly usable in the context of business re-engineering, because the complete approach assumes the existence of a service (otherwise there is no notion of perceived delivery). This is at first sight not useful for the exploration and design phase of a value web, but certain elements of the SERVQUAL instrument are usable. In Figure 3 for instance value object $a$ represents the service under consideration. In case business planners are interested in meeting end customer expectations of service quality, the first questionnaire dealing with the customer expectations builds a sufficient basis. Possible end consumers, like e.g. internet surfers, are asked to state subjectively their qualitative expectations on a potential business service. The results of this questionnaire build an essential 
basis for meeting quality expectations at the end consumption point in networked businesses. Note that the SERVQUAL instrument stems from a time when most business services were traditional services like a haircut or a taxi drive. With the advent of the internet, thus of commercial IT services, many slightly altered SERVQUAL versions have been introduced, like for example E-S-QUAL (Parasuraman, 2005) or IS-SERVQUAL (James, 2002), which take the new internet environment into account.

\subsection{Planning Quality Properties of Value Object Production/Composition}

The second quality issue $\mathrm{B}$, the qualitative planning of value object production/composition, is also an important quality issue with respect to networked value constellations. $e^{3}$-value models often only show trading path of value objects, but sometimes a value object needs to be composed or produced at one of the actors. In figure 4, the AND join at the producer indicates that the producer composes out of value objects $e$ and $g$, a new value object $c$, which is later transferred further to the retailer.

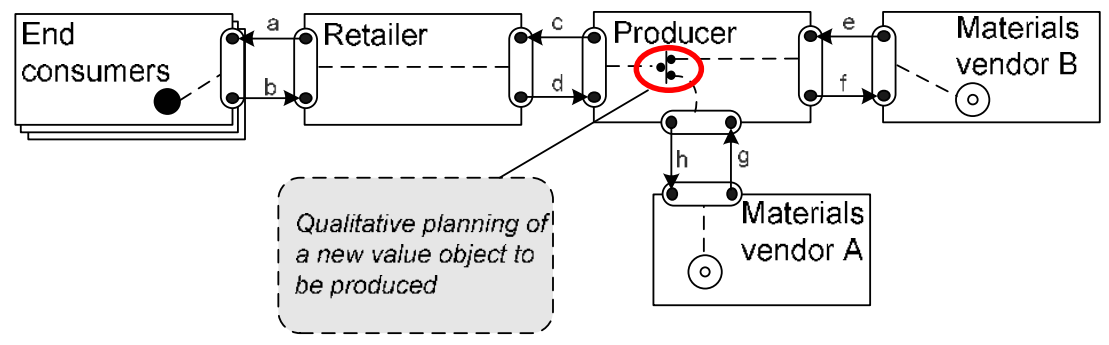

Figure 4 - Qualitative Planning of Value Object Production

The $e^{3}$-value developers state that a value object can be a good, a service, or even an experience (Gordijn, 2001). In case it is a physical good, the quality function deployment (QFD) tool, sometimes also called the House of Quality, can be used for planning in a qualitative way. QFD originated in the early 1970s in Japan in the automotive industry (Hauser, 1988). Its usage was fast expanded to quality planning of other tangible goods than automobiles and is generally applicable to this category. The next category, services, also profits from QFD, since its usage was adapted in the late 1990s for qualitative service design (Ermer, 1998). The last category, experience, is somehow fuzzy, as far as it can indicate to experience something new or to be satisfied for whatever reason. In some cases this experience is not semantics, like for instance music. We do not consider this fuzzy category implementable in terms of information technology.

\subsection{Planning Quality Properties of Value Object Transfer}

Every value object transfer has to be planned also in a qualitative way. Considering the value object transfers in the figure below, we can assume that value objects $b, d$, $f$, and $h$ represent money or fee in terms of the obligation to refund a value object. It is clear that such payments need to be secure, reliable and fast. Apart from that, we are not aware of any technique that deals with the quality of value object transfers, 
so that this represents an unresolved problem. Again, QFD seems a good candidate for performing this task. In future research we will investigate its usage and define heuristics with respect to planning qualitative value object transfers. SERVQUALlike approaches might also be good candidates, as the transfer of a value object is itself a service.

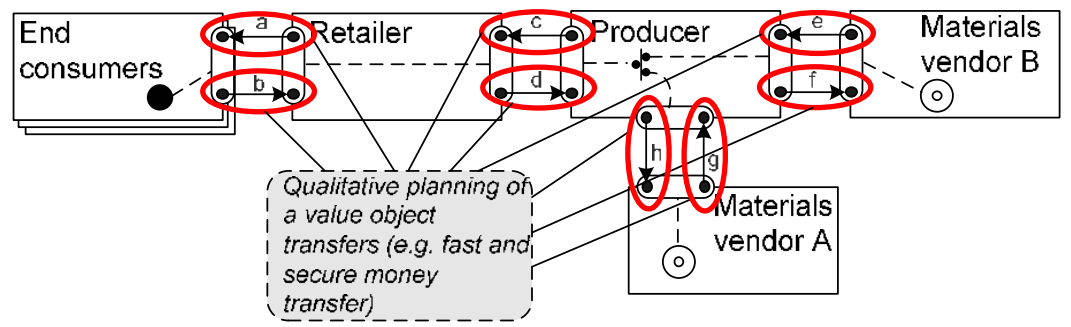

Figure 5 - Qualitative Planning of Value Object Transfers

\section{QUALITY ISSUES AT BOTH LEVELS}

In $e^{3}$-value we represent a business network as a graph, where we indicate which business node delivers which value objects to whom and what it gets in return. The IOS landscape of a networked value constellation is usually also represented as a graph, connecting the information systems with each other.

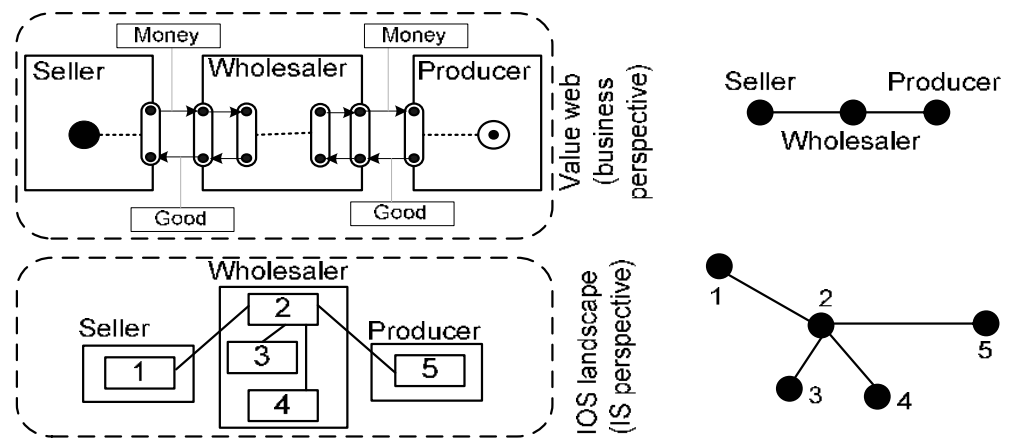

Figure 6 - Investigating underlying graphs.

There exists many situations that can lead to changes of the business network, thus of the underlying graph. For instance, after some time of existence one business actor in the networked value constellation decides to outsource certain activities to a new business actor. As a consequence, one business actor node has to be added to the representation, which means in turn that we are dealing with a new graph. Changes of networked value constellations are also conceivable during the ebusiness exploration phase, while deconstructing and reconstructing value models. Kumar and van Dissel (1996) argue that inter-organizational dependency patterns, previously identified by Thompson (1967), are as such to be reflected by the design of IOS. Figure 6 shows that this is not a one-to-one mapping or derivation, which is reflected by the underlying graphs on the right. The wholesaler hosts for instance three information systems. Current work (Zarvić, 2008b) gives evidence that it is important to consider structural properties of the underlying graphs as indicators of 
certain quality aspects that arise while opting for one network constellation or another. In one of our case studies, we showed that an outsourcing option has lead on the one hand to a less complex network, but on the other hand also to a less reliable constellation (Zarvić, 2008b). Take for instance figure 6, where we deal with a chained pattern style at the business perspective, but at the IS perspective we have a combination of chained and pooled pattern style. For the discussion of complexity and reliability issues, our simple approach, which is based on basic properties from graph theory, has to be applied to both perspectives.

\section{QUALITY ISSUES OF UNDERLYING SOFTWARE}

Quality issue E, namely the quality of software-intensive systems (IS perspective), is a well researched area, where we can make use of many existing software quality models, or even change and update them for fitting our purposes (Lauesen, 2002). In figure 7 the six sets of characteristics, including subcharacteristics, of the ISO 9126 quality model are shown. ISO 9126 is not only useful for the evaluation, but also for the specification of quality (Stefani, 2008). As far as the functions/services of software products are the artifacts that enable and support value object transfers, the external metrics suggested by ISO 9126 could be used as the technical means for analyzing the quality of value object transfers at IS level (see Section 3.3). This is a clear interrelation and represents a part of the described future research in section 3.3.

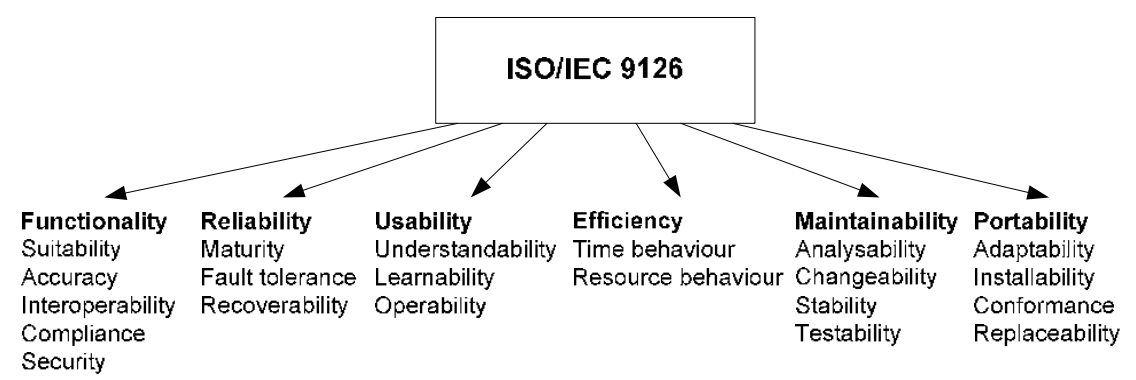

Figure 7 - Characteristics and sub-characteristics of ISO 9126.

\section{SUMMARY AND CONCLUSIONS}

We have presented a conceptual framework for business-IT alignment that includes next to functional also quality issues. More precisely, these quality issues were situated along the business perspective and the IS perspective, tools for dealing with them were allocated, and interrelated. Thereby we indicated which issues represent resolved and which unresolved problems. Next to the fact that we described the need for quality during the planning and alignment process in a comprehensive way, it should be noted that solely two perspectives were considered. This means that other conceivable perspectives, like e.g. a process or workflow perspective or even a hardware perspective, were not considered in this paper. Summing up, quality issues $\mathrm{A}, \mathrm{B}$ and $\mathrm{D}$ are not unresolved issues, as far as existing techniques can be used to 
tackle them. However, quality issues $\mathrm{C}$ and $\mathrm{E}$ are highly interrelated and represent unsolved research problems that we aim to approach in future research. Also other instruments like the goal-question-metric (GQM) are conceivable in this context, but they do not offer a possibility to prioritize technical means as e.g. QFD does. However, this is not decreasing the value of the present work, as far as it represents, to the best of our knowledge, the first systematic attempt to view and analyze quality from a total quality management (TQM) point of view for the respective perspectives. In future work we aim to further investigate the QFD approach and its applicability to the value web context. In particular we intend to define heuristics for its usage with respect to qualitative value object transfers and their realization at the software level by considering software characteristics and metrics as suggested by ISO 9126.

\section{REFERENCES}

1. Derzsi Z, Gordijn J., van Eck P. "Assessing Feasibility of IT-enabled Networked Value Constellations: A Case Study in the Electricity Sector". In Proceedings of $19^{\text {th }}$ International Conference (CAiSE 2007), Trondheim, Norway, Springer LNCS 4495: 66-80, 2007.

2. Ermer D, Kniper M. Delighting the customer: Quality function deployment for quality service design. Total Quality Management 1998; 9(4\&5): 86-91.

3. Gordijn J, Akkermans H. Designing and Evaluating E-Business Models. IEEE Intelligent Systems 2001; 16(4): 11-17.

4. Gordijn J, Akkermans H. Value-based requirements engineering: exploring innovative e-commerce ideas. Requirements Engineering Journal 2003; 8(2): 114-134.

5. Hauser J, Clausing D. The House of Quality. Harvard Business Review May-June 1988; 63-73.

6. ISO 8402. Quality Management and Quality Assurance - Vocabulary. International Organization for Standardization, April 1994.

7. ISO 9126. Information Technology - Software Quality Characteristics and Metrics (ISO/IEC 9126-1 and ISO/IEC 9126-2). International Organization for Standardization, June 1995.

8. Jiang J, Klein G, Carr CL. Measuring Information System Service Quality: SERVQUAL from the other side. MIS Quarterly 2002; 26(2): 145-166.

9. Kumar K, van Dissel H. Sustainable Collaboration: Managing Conflict and Cooperation in Interorganizational Systems. MIS Quarterly 1996; 20(3): 279-300.

10. Lauesen S. Software Requirements - Styles and Techniques. Addison Wesley 2002.

11. O'Sullivan J, Edmond D, ter Hofstede A. What's in a Service? Towards Accurate Description of NonFunctional Service Properties. Distributed and Parallel Databases 2002; 12: 117-133.

12. Parasuraman A, Zeithaml VA, Berry L. A Conceptual Model of Service Quality and Its Implications for Future Research. Journal of Marketing 1985, 49: 41-50.

13. Parasuraman A, Zeithaml VA, Malhotra A. E-S-QUAL: A Multiple-Item Scale for Assessing Electronic Service Quality. Journal of Service Research 2005, February, 213-233.

14. Stefani A, Xenos M. E-commerce system quality assessement using a model based on ISO 9126 and Belief Networks. Software Quality Journal 2008, 16:107-129.

15. Thompson JD. Organizations in Action. McGraw-Hill 1967.

16. Zarvić N, Wieringa RJ, van Daneva M. "Towards Information Systems Design for Value Webs". In Proceedings of Workshops and Doctoral Consortium of CAiSE 2007, Trondheim, Norway, Tapir Academic Press, 453-460, 2007.

17. Zarvić N, Wieringa RJ, van Eck P. "Checking the Alignment of Value-based Business Models and IT Functionality". In Proceedings of $23^{\text {rd }}$ Annual ACM Symposium on Applied Computing, Fortaleza, Brazil, (accepted for publication) 2008.

18. Zarvić N. "Considering Structural Properties of Inter-organizational Network Fragments during Business-IT Alignment”. (submitted) 2008. 